\title{
Online Diagnosis of State of Health for Lithium-ion Batteries Based on Short-Term Charging Profiles
}

\author{
Xing Shu ${ }^{1}$, Guang $\mathrm{Li}^{2}$, Yuanjian Zhang ${ }^{3}$, Jiangwei Shen ${ }^{1}$, Zheng Chen ${ }^{1,2^{*}}$ and Yonggang $\mathrm{Liu}^{4 *}$ \\ ${ }^{1}$ Faculty of Transportation Engineering, Kunming University of Science and Technology, Kunming, 650500, China \\ ${ }^{2}$ School of Engineering and Materials Science, Queen Mary University of London, London, E1 4NS, United Kingdom \\ ${ }^{3}$ Sir William Wright Technology Center, Queen's University Belfast, Belfast, BT9 5BS, United Kingdom \\ ${ }^{4}$ State Key Laboratory of Mechanical Transmissions \& School of Automotive Engineering, Chongqing University, \\ Chongqing, 400044, China \\ Email: shuxing92@kust.edu.cn, g.li@qmul.ac.uk,y.zhang@qub.ac.uk, shenjiangwei6@163.com, chen@kust.edu.cn, \\ andylyg@umich.edu \\ Corresponding Author: Zheng Chen (chen@kust.edu.cn) and Yonggang Liu (andylyg@umich.edu)
}

Abstract: In this study, a machine learning method is proposed for online diagnosis of battery state of health. A prediction model for future voltage profiles is established based on the extreme learning machine algorithm with the short-term charging data. A fixed size least squares-based support vector machine with a mixed kernel function is employed to learn the dependency of state of health on feature variables generated from the charging voltage profile without preprocessing data. The simulated annealing method is employed to search and optimize the key parameters of the fixed size least squares support vector machine and the mixed kernel function. By this manner, the proposed algorithm requires only partial random and discontinuous charging data, enabling practical online diagnosis of state of health. The model training and experimental validation are conducted with different kernel functions, and the influence of voltage range and noise are also investigated. The results indicate that the proposed method can not only maintain the state of health estimation error within $2 \%$, but also improve robustness and reliability.

Key Words: state of health; voltage prediction; mixed kernel function; fixed size least squares support vector machine; lithium-ion battery

\section{INTRODUCTION}

Lithium-ion batteries have been considered as a mainstream solution of storing electrical energy and have been popularly used as energy storage elements in electric vehicles (EVs) due to their long lifespan, fast charging capability and high energy density, compared to other energy storage media [1]. However, the performances of lithium-ion batteries will be gradually deteriorated with operation [2]. Knowledge of current health status is imperative to guarantee reliable safe operation of lithium-ion batteries [3]. State of health (SOH), 
which often gives the percentage value of capacity degradation and/or internal resistance increase, can supply valuable references to indicate healthy status of batteries. In addition, $\mathrm{SOH}$ is also crucial for estimation of state of charge (SOC) and state of energy (SOE) [4]. Nonetheless, SOH estimation is difficult, especially in EV applications, to be directly measured or counted, as full charge/discharge is quite time-consuming and difficult to implement. In this context, a body of researches have been spurred to achieve SOH diagnosis based on advanced algorithms [5]. Generally, these $\mathrm{SOH}$ prediction methods can be categorized into three types: direct measurement/calibration, machine learning approaches and adaptive algorithm-based methods.

The measurement/calibration approaches obtain $\mathrm{SOH}$ through specially designed experiments, such as the electrochemical impedance spectroscopy (EIS) test, coulomb counting calibration and hybrid pulse power characterization (HPPC) test. As the battery's impedance increases with degradation, EIS is commonly used to account for impedance variation, thus supplying an effective index for aging diagnosis [6]. HPPC test can contribute to identification of open circuit voltage (OCV) and internal resistance, thereby establishing a nonlinear relationship between internal resistances or OCV and SOH [7]. The coulomb counting method simply discharges the battery to empty, or fully charges the battery from empty, and then SOH can be calculated by dividing the released/accumulated capacity over the nominal value [8]. Unfortunately, the direct measurement approaches entail special current excitation and long duration. Obviously, they are more suitable under laboratory conditions, rather than applications in real scenarios.

Adaptive algorithm-based approaches have been successfully exploited in the literature. Their estimation processes mainly involve model establishment and application of adaptive algorithms. To achieve $\mathrm{SOH}$ estimation, an efficient model characterizing battery's degradation performance is usually indispensable, like equivalent circuit models (ECM) and semi-empirical models. On this basis, advanced adaptive algorithms are synchronously employed to track the deterioration of battery capacity. Ref. [9] leverages a dual H-infinity filter (DHIF) in a multi-scale framework to monitor battery SOC and capacity online. In [10], a forgetting factor recursive expanded least square method is proposed to identify the OCV of the built fractional order model and the battery capacity is recognized through incremental capacity analysis (ICA). In [11], two extended Kalman filters (EKFs) are incorporated to calibrate SOC microscopically and update SOH macroscopically. In addition, other adaptive algorithms, such as slide mode observer [12], particle filter (PF) [13] and Lyapunovbased adaptive observer [14], are also investigated for SOH estimation. These algorithms can be implemented 
in different loading scenarios without specially designed current profiles; in other words, fixed current tests are usually not required. These methods can intuitively describe the physical relationship between battery parameters and intrinsic aging processes [15]. Nevertheless, their precision and adaptability are susceptive to noise and operating conditions.

Due to less dependence on physical insights and deterministic characteristic models, machine learning approaches have progressively emerged to achieve $\mathrm{SOH}$ estimation [16], including Gaussian process regression (GPR) [17], support vector machine (SVM) [18], neural network (NN) [19] and relevance vector machine (RVM) [20]. These methods usually find hidden variation laws and statistical information between $\mathrm{SOH}$ and measured voltage, current and temperature data during charge/discharge process. To diagnose battery $\mathrm{SOH}$ by means of machine learning methods, an overarching task is to process the measured data and derive representative features to highlight aging variation. In [21], four geometrical parameters are generated from battery cycling processes, including the length of constant voltage (CV) charging mode, the maximum curvature radius of $\mathrm{CV}$ profile, the area under the $\mathrm{CV}$ stage and the maximum slope of the early stage discharge voltage profile. Ref. [22] employs the RVM to map the nonlinear relationship between $\mathrm{SOH}$ and five variables, i.e., the final charge current, the initial charge voltage, the final charging voltage, the charging capacity during the CV stage and the constant current (CC) stage capacity. The reported health feature extraction methods can greatly contribute to the estimation of battery $\mathrm{SOH}$. Nevertheless, these methods often entail full cycling experiment, which is seldom encountered in engineering practice. To cope with it, $\mathrm{SOH}$ calibration is fulfilled by partial discharge/charge voltage curves [23]. In [24], a small portion of data extracted from the CC charging process is selected as the inputs of the GPR model to attain capacity estimation. In [25], the peak and valley of the incremental capacity and differential voltage (DV) are prudentially detected as the feature inputs of three different regression methods: SVM, NN and linear regression. The $\mathrm{SOH}$ prediction is extensively investigated based on partial charging segments; however, the initial and terminal voltages of these features are fixed, and the battery needs to be charged across the initial and terminal voltages in these algorithms [26]. In real applications, the battery charging behavior is often a stochastic process, and end-users may start and stop charging randomly. From this point of view, SOH estimation based on full charge or fixed voltage range is usually infeasible in practice. 
Additionally, performances of $\mathrm{SOH}$ estimation methods are also related to selected algorithms. Among all the existing machine learning algorithms, SVM can construct a multidimensional nonlinear relationship with a small training set [20]. Furthermore, it is insensitive to the dimension of test data [18]. In [27], a hybrid method incorporating SVM and differential evolution (DE) is introduced to forecast the remaining useful life (RUL), and the DE is furnished to explore the optimal parameters of SVM. Through the established OCV and Thevenin models, Ref. [28] employs the least squares-SVM (LS-SVM) algorithm to achieve reliable SOH prediction. However, existing SVM based methods, to the best of authors' knowledge, still do not optimize the prediction performance when they are employed to estimate $\mathrm{SOH}$ of lithium-ion batteries. We find that, when diagnosing SOH of lithium-ion batteries, most of the existing SVM methods in literature employ only traditional kernel functions, which usually lose generalization for ensuring the estimation accuracy. In other words, the benefits of single kernel function still need to be improved for better SOH estimation. It may be a promising way to upgrade the single kernel function knowledge to a mixed kernel function, thereby merging both advances of two single kernel functions and enhancing the estimation performance of SVM in terms of estimation accuracy and generalization ability.

For the sake of filling the gap of research, we devoted our contribution to developing an online $\mathrm{SOH}$ diagnosis algorithm for lithium-ion batteries based on short-term random discontinuous charging data. In particular, an advanced voltage prediction approach is developed based on the extreme learning machine (ELM) algorithm, which is proved to be a quick manner to train and learn inherent laws of voltage variation. An effective online SOH diagnosis method is presented based on the fixed size LS-SVM. We accentuate the significance of opting for proper kernel functions, which are often neglected in previous studies, and the benefits of applying mixed kernel functions. In addition, to prevent inaccurate diagnosis incurred by inappropriate parameters of fixed size LS-SVM, the simulated annealing (SA) algorithm is exploited to optimize the algorithm parameters. The validation results demonstrate that the proposed method can effectively diagnose the $\mathrm{SOH}$ with less than $2 \%$ error.

The rest of this study is structured in the following manner: Section II discusses the fundamental knowledge of ELM and fixed size LS-SVM. The overall procedures of voltage forecast and SOH estimation for lithium-ion batteries are described in Section III. Section IV presents the experiment processes and analyzes the validation results. The main conclusions are drawn in Section V. 


\section{Methodology}

The goal of this study is to find a machine learning method to estimate the battery SOH based on shortterm charging data. To achieve a robust design, we assume the data are randomly selected and endeavor to predict the complete charging voltage curve from the short-term data by the ELM algorithm. On this basis, the feature variables are extracted, and the SVM is employed to learn the mapping function from the inputs to SOH. In this section, the principles of ELM and fixed size LS-SVM techniques are elaborated.

\section{A. Extreme Learning Machine}

The ELM has been extensively investigated because of its single hidden layer structure and fast learning speed [29]. These characteristics can well meet the requirement of voltage online training and prediction. Additionally, one only needs to set the number of neurons of ELM, since the input weight and the threshold of hidden layer are generated randomly [30]. For a data set containing $n$ samples $\left(\boldsymbol{x}_{i}, \boldsymbol{y}_{i}\right)$, where $\boldsymbol{x}_{i} \in R^{n}$ represents the input and $\boldsymbol{y}_{i} \in R^{n}$ denotes the corresponding desired output, the output of the ELM can be formulated as:

$$
\boldsymbol{t}_{j}=\sum_{i=1}^{N_{\text {nauron }}} \beta_{i} g\left(\boldsymbol{\omega}_{i} \cdot \boldsymbol{x}_{j}+b_{i}\right), \quad j=1,2, \ldots, n
$$

where $\omega_{i}=\left[\omega_{i 1}, \omega_{i 2}, \cdots, \omega_{i n}\right]^{T}$ denotes the weight vector connecting the input neurons and the $i$ th hidden neuron, $b_{i}$ represents the bias of the $i$ th hidden neuron, $g(\cdot)$ is activation function, $\beta_{i}=\left[\beta_{i 1}, \beta_{i 2}, \cdots, \beta_{i m}\right]^{T}$ denotes the weight vector corresponding to the $i$ th hidden neuron and the output neurons, and $N_{\text {neuron }}$ represents the number of neurons. The purpose of ELM is to find the optimal output weight quickly and minimize the output error. The objective function can be formulated as:

$$
\sum_{i=1}^{N_{\text {neuron }}}\left\|y_{i}-t_{i}\right\|=0
$$

It means there exist $\beta_{i}, \omega_{i}$ and $b_{i}$, such that

$$
\boldsymbol{y}_{j}=\sum_{i=1}^{N_{\text {neuron }}} \beta_{i} g\left(\boldsymbol{\omega}_{i} \cdot \boldsymbol{x}_{j}+b_{i}\right), \quad j=1,2, \ldots, n
$$

Equation (3) can be rewritten as:

$$
\boldsymbol{H} \beta=\boldsymbol{y}
$$


where $\beta=\left[\beta_{1}, \beta_{2}, \ldots, \beta_{N_{\text {neuron }}}\right]^{T}$, and $\boldsymbol{y}=\left[y_{1}, y_{2}, \ldots, y_{n}\right]^{T} . \boldsymbol{H}$ denotes the hidden layer output matrix and can be written as:

$$
\boldsymbol{H}\left(\omega_{1}, \ldots \omega_{N_{\text {neuron }}}, x_{1}, \ldots x_{n}, b_{1}, \ldots b_{N_{\text {neuron }}}\right)=\left[\begin{array}{lll}
g\left(\omega_{1} \cdot x_{1}+b_{1}\right) & \ldots & g\left(\omega_{N_{\text {neuron }}} \cdot x_{1}+b_{N_{\text {neuron }}}\right) \\
\vdots & \ldots & \vdots \\
g\left(\omega_{1} \cdot x_{n}+b_{1}\right) & \ldots & g\left(\omega_{N_{\text {neuron }}} \cdot x_{n}+b_{N_{\text {neuron }}}\right)
\end{array}\right]
$$

In the ELM model, $\omega_{i}$ and $b_{i}$ can be chosen randomly. The optimal solution can be obtained, as:

$$
\hat{\beta}=\boldsymbol{H}^{+} \boldsymbol{Y}
$$

where $\boldsymbol{H}^{+}$denotes the Mooree-Penrose generalized inverse of $\boldsymbol{H}$. Generally, the number of neurons is less than that of training samples, i.e., $N_{\text {neuron }}<n$. Here, the least squares is applied to reformulate (6), as:

$$
\hat{\beta}=\left(\boldsymbol{H}^{T} \boldsymbol{H}^{-1}\right) \boldsymbol{H}^{T} \boldsymbol{Y}
$$

Based on the preparation work, the optimal ELM will be constructed. It is apparent that any iteration process is not involved, and this advanced manner brings obvious improvement of the modeling speed, which satisfies the demand of fast online prediction of future voltage. Subsequently, the fixed size LS-SVM will be investigated.

\section{B. Fixed Size LS-SVM}

SVM stems from statistical theory and is widely implemented in data forecasting and classification. Until now, SVM has been developed with different extensions, such as LS-SVM [31], fuzzy SVM [32] and Proximal SVM (PSVM) [33]. Amongst them, the fixed size LS-SVM can not only improve sparseness but also enhance robustness. For this reason, the fixed size LS-SVM is employed and investigated for SOH estimation in this study. Similar to ELM, we consider a data set containing $n$ samples $\left(\boldsymbol{x}_{\boldsymbol{i}}, \boldsymbol{y}_{\boldsymbol{i}}\right)$, and the SVM function is designated as:

$$
\boldsymbol{y}_{i}=w^{T} \phi\left(\boldsymbol{x}_{i}\right)+b_{S V M}
$$

where $\boldsymbol{y}_{i}$ stands for the output value, $\phi(\cdot)$ is a mapping function, and $w \in R^{n}$ and $b_{S V M} \in R^{n}$ represent the weight and intercept, respectively. The target of SVM is to learn a mapping function such that the maximum divergence between the output obtained from the training set and the real value is less than a preset threshold. Thus, the optimal problem can be formulated as: 


$$
\left\{\begin{array}{l}
\min Q(w, \boldsymbol{e})=\frac{1}{2}\|w\|^{2}+\frac{\gamma}{2} \sum_{i=1}^{n} e_{i}^{2} \\
\text { s.t. } y_{i}=w \cdot \phi\left(x_{i}\right)+b_{S V M}+e_{i}, i=1,2, \ldots, n
\end{array}\right.
$$

where $\boldsymbol{e}=\left[e_{1}, e_{2}, \ldots, e_{n}\right]^{T}$. By introducing the Lagrange multiplier, a series of inequalities in the standard SVM is replaced by a group of linear constraints, and the problem can be translated into an equivalent unconstraint problem, as:

$$
L_{1}\left(w, b_{S V M}, \boldsymbol{e}, \boldsymbol{\alpha}\right)=\frac{1}{2}\|w\|^{2}+\frac{\gamma}{2} \sum_{i=1}^{n} e_{i}^{2}-\sum_{i=1}^{n} \alpha_{i}\left(w \cdot \phi\left(x_{i}\right)+b_{S V M}+e_{i}-y_{i}\right)
$$

where $\boldsymbol{\alpha}=\left[\alpha_{1}, \alpha_{2}, \ldots, \alpha_{n}\right]$, and $\boldsymbol{e}=\left[e_{1}, e_{2}, \ldots, e_{n}\right]$. By finding the partial derivative of $(10)$ and setting the result to zero, we can attain:

$$
\left\{\begin{array}{l}
\frac{\partial L}{\partial w}=0 \Rightarrow \omega=\sum_{i=1}^{n} \alpha_{i} \phi\left(x_{i}\right) \\
\frac{\partial L}{\partial b_{S V M}}=0 \Rightarrow \sum_{i=1}^{n} \alpha_{i}=0 \\
\frac{\partial L}{\partial e_{i}}=0 \Rightarrow \alpha=\gamma e_{i} \\
\frac{\partial L}{\partial \alpha_{i}}=0 \Rightarrow b_{S V M}=y_{i}-\omega^{T} \phi\left(x_{i}\right)-e_{i} \\
i=1,2, \ldots, n
\end{array}\right.
$$

By solving (10), the following nonlinear mapping model can be yielded, as:

$$
y(x)=\sum_{i=1}^{n} \alpha_{i} K\left(x, x_{i}\right)+b_{S V M}
$$

where $K(\cdot)$ represents the kernel function. Note that the support vectors of SVM are selected randomly, and we do not give any criteria to choose the optimal support vectors. Obviously, it needs to be improved. With this in mind, the arbitrary entropy is introduced to find the optimal support vectors [34], as:

$$
H_{R}=-\lg \int p(x)^{2} d x
$$

where $p(x)$ represents the density distribution of $x$, and

$$
\int p(x)^{2} d x \approx \int \hat{p}(x) d x=\frac{1}{M^{2}} \sum_{i=1}^{M} \sum_{j=1}^{M} K\left(x_{i}, x\right)
$$

where $M$ denotes the number of support vectors, Consequently, equation (13) can be solved, as: 


$$
H_{R} \approx-\lg \left(\frac{1}{M^{2}} \sum_{i=1}^{M} \sum_{j=1}^{M} K\left(x_{i}, x\right)\right)
$$

To reach the full potential of fixed size LS-SVM, it is crucial to select or design a suitable kernel function. Indeed, there are a number of kernel functions; nonetheless, it is a case-by-case issue to select a suitable kernel function when facing different problems. Currently, these kernel functions can be roughly classified into two groups: global kernel functions and local ones. The radial basis kernel function (RBF), as described in (16), is one representative of the former, and one typical local kernel function is the polynomial kernel function, which is defined in (17). Global kernel functions usually feature powerful generalization capabilities but lead to fragile learning capability, which is opposite to those of local kernel functions [35]. A mixed kernel function can merge two or more single kernel functions and take advantage of assorted kernel functions [36]. Based on the Mercer theory [37], a new kernel function, as formulated in (18), is designed with the linear combination of different kernel functions.

$$
\begin{gathered}
K_{R B F}\left(x_{i}, x_{j}\right)=\exp \left(-\left\|x_{i}-x_{j}\right\|^{2} /\left(2 \sigma^{2}\right)\right) \\
K_{\text {Poly }}\left(x_{i}, x_{j}\right)=\left(x_{i} \cdot x_{j}+t\right)^{q} \\
K_{\text {Mix }}=\lambda_{1} K_{\text {Poly }}+\lambda_{2} K_{R B F}
\end{gathered}
$$

where $\lambda_{1}$ and $\lambda_{2}$ denote the weights of polynomial function and RBF, respectively. Moreover, $\lambda_{1}+\lambda_{2}=1$. When $\lambda_{1}=1$, the mixed kernel function degenerates to the conventional polynomial kernel function, and when $\lambda_{2}=1$, the mixed kernel function is a standard RBF. In addition, in spite of fixed size LS-SVM or that with mixed kernel functions, their function parameters all need to be determined, and the process will be elaborated in the next section.

\section{SOH DIAGNOSIS BASED ON MEASURABLE PARAMETERS}

In this section, the aging experiment conducted in our laboratory is elaborated, and the process of feature variable generation is investigated, followed by the implementation process of proposed voltage prediction approach. Additionally, the overall framework of the proposed $\mathrm{SOH}$ diagnosis algorithm is presented and discussed. 


\section{A. Aging Experiment}

In this study, three nickel-cobalt-manganese (NCM)/graphite batteries, labeled as Bat. 1, Bat. 2 and Bat. 3 , are experimented in our laboratory to acquire the aging data. The nominal voltage and capacity of cells are 3.6 $\mathrm{V}$ and $4 \mathrm{Ah}$, respectively; and the operating voltage ranges from $3 \mathrm{~V}$ to $4.15 \mathrm{~V}$. Here, the $\mathrm{CC}-\mathrm{CV}$ charging scheme and $\mathrm{CC}$ discharge schedule are repetitively operated. The charging and discharging rates are respectively set to $0.5 \mathrm{C}$ and $1 \mathrm{C}$, where $\mathrm{C}=4$ indicates the rated capacity value of battery. The $\mathrm{CC}-\mathrm{CV}$ mode stops until the voltage reaches $4.15 \mathrm{~V}$ and meanwhile the charging current decreases to $0.02 \mathrm{C}$. The discharge experiment is terminated when the voltage decreases to $3 \mathrm{~V}$. In addition, two shelving operations with the length of 5 minutes are imposed when switching between charging and discharging. Note that all cycle tests are conducted at room temperature, and the temperature influence is not taken into account in this study.

\section{B. Aging Features Generation based on Measurable Parameters}

To diagnose battery $\mathrm{SOH}$ accurately, first, it is imperative to determine the feature variables that can reliably reveal the deterioration trend of lithium-ion batteries. Given the calculation and error of voltage prediction, these features should be easily obtained and characterize strong anti-interference capability. Due to the inherent complexity of battery electrochemical model and its complicated inner structure, it is difficult to capture effective features in a static manner. In this context, degradation characteristics of batteries are often highlighted by external measurements variation during charging/discharging processes. Since discharging current profiles are usually stochastic, leading to difficulty of extracting a suitable feature variable. In contrast, the charging process is more regular and easier to be investigated. One example of charging voltage curves under different cycle numbers is depicted in Fig. 1. It can be clearly found that with the degradation of battery, the $\mathrm{CC}$ charging time decreases gradually. The fresh battery takes 64 minutes when charging from $3.45 \mathrm{~V}$ to $3.8 \mathrm{~V}$, while the battery after cycled 500 times and 900 times merely last about 42 minutes and 27 minutes, respectively, only occupying $65.6 \%$ and $42.2 \%$ of that of the fresh battery. Similarly, a period of 25.5 minutes is required for a new battery when charging from $3.8 \mathrm{~V}$ to $4 \mathrm{~V}$. In contrast, it takes only 24 minutes to obtain the same voltage increment for the 900 times cycled battery. 


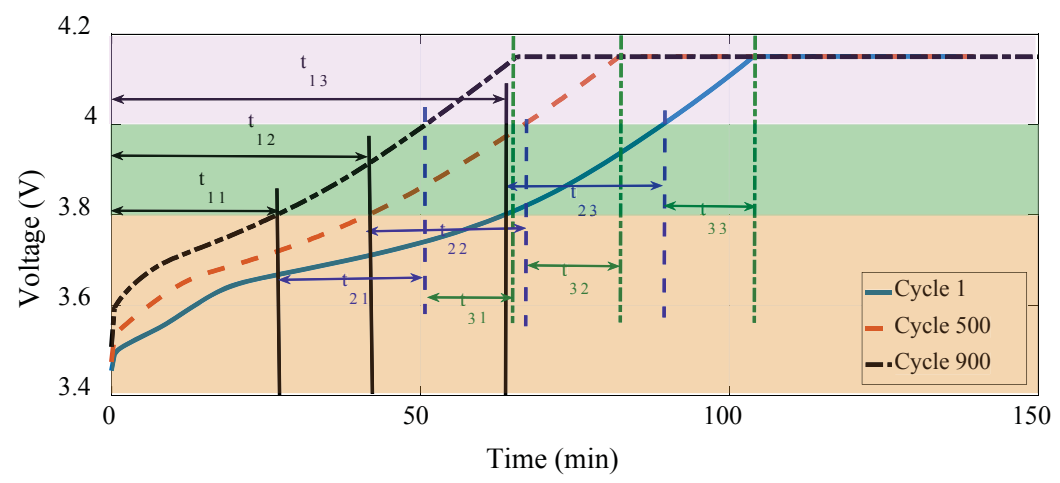

Fig. 1. The CC-CV charging sketch at different cycles.

These phenomena indicate that the variation among voltage profiles after cycling different times can be exerted to characterize the aging behaviors of batteries. As the battery ages, the time required to charge from one voltage to another value gradually decreases. In other words, the duration decrement of the same voltage increase interval can intentionally be addressed to quantify the battery SOH variation. As such, the charging duration from $3.4 \mathrm{~V}$ to $3.8 \mathrm{~V}, 3.8 \mathrm{~V}$ to $4 \mathrm{~V}$ and $4 \mathrm{~V}$ to $4.15 \mathrm{~V}$ are chosen as the basic features for $\mathrm{SOH}$ diagnosis, and the corresponding voltage threshold is labeled as $V_{l}=3.4 \mathrm{~V}, V_{m 1}=3.8 \mathrm{~V}, V_{m 2}=4 \mathrm{~V}$ and $V_{h}=4.15 \mathrm{~V}$. Furthermore, the influence of voltage range selection will be discussed in Section IV.

\section{Voltage Prediction}

The substance of voltage prediction is to forecast the whole voltage response curve corresponding to the future charging current. In fact, this is not a challenging task in the CC charging process, as the current is known during this process. We assume that the battery has been charged for a short period $t$, and the recorded current, voltage, temperature and SOC, referred to as $I_{a}, V_{a}, T_{a}$ and $s_{a}$, are collected from beginning to the present moment for voltage forecast. All of these values can be random and discontinuous, nonetheless they should be one-to-one correspondence; in other words, when current is collected at step $k$, the voltage, SOC and temperature should also be acquired at this time. Our goal is to predict the partial voltage values without measure and therefore acquire the whole charging voltage information using the available data. To attain it, the ELM algorithm is applied, and a data set $\left\{\left(\operatorname{In}_{1}, O V_{1}\right),\left(I n_{2}, O V_{2}\right), \cdots,\left(I n_{n}, O V_{n}\right)\right\}$ employed to train the model is provided, where $I n_{i}$ denotes the input vector at time step $i$, and $O V_{i}$ is the actual voltage corresponding to $I n_{i}$. The available data at time step $i$ are stored in an input vector, as

$$
I n_{i}=\left[I_{i}, T_{i}, s_{i}\right]
$$


The ELM model is trained by the measured voltage data segment, and then the trained model is implemented to predict the remaining unknown voltage data. By this manner, the complete voltage charging curve can be achieved.

\section{State of Health Diagnosis}

The overall framework of SOH diagnosis based on short-term charging data is depicted in Fig. 2. The proposed approach contains three modules: voltage prediction, feature extraction, fixed size LS-SVM modeling and estimation. First, the proposed ELM algorithm is exploited to constitute a voltage prediction model for forecasting the complete charging curve according to the known partial information. Then, the output data of ELM are disposed, and the features of SOH are extracted. Finally, the fixed size LS-SVM algorithm is furnished to construct the nonlinear mapping among the input feature variables and $\mathrm{SOH}$, thereby accomplishing the SOH diagnosis by serviceable model learning. In addition, the fixed size LS-SVM training module includes a crucial parameter optimization step. There are six parameters in total that need to be set $a$ priori in the algorithm and the included mixed kernel function, i.e., the penalty factor $\gamma$, the RBF kernel parameter $\sigma$, the polynomial parameters $q$ and $t$, and the weights of RBF and polynomial kernel function $\lambda_{1}$ and $\lambda_{2}$. The proposed parameter optimization problem is strongly nonlinear, enabling it difficult to find the optimal parameters through numeric methods. To solve it, the SA algorithm is implemented to find them effectively [38]. The SA algorithm chooses the six parameters as the control target $c=\left(\begin{array}{lll}\sigma, q, & \gamma, \lambda_{1}, \lambda_{2}\end{array}\right)$. Meanwhile, the mean absolute error (MAE) between the output $\mathrm{SOH}$ and real value is set as the evaluation criterion. The update of theses variables conforms to the probability distribution. When the termination conditions are met, the algorithm will stop and finally give the optimal $c$. Note that in this study, the optimal fixed size LS-SVM model with a mixed kernel function is trained. 


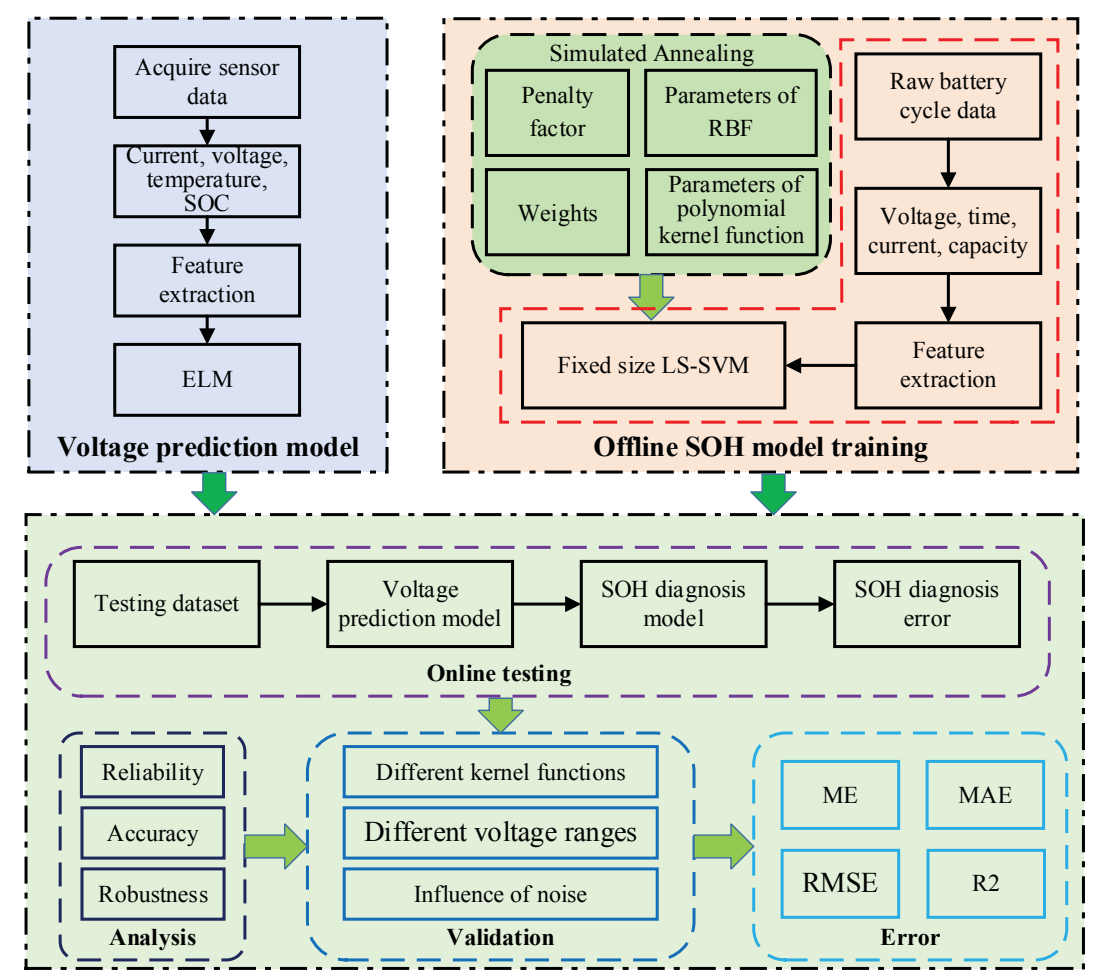

Fig. 2. Overall flowchart of the proposed $\mathrm{SOH}$ diagnosis.

\section{EXPERIMENTAL RESULTS AND DISCUSSION}

In this section, the mixed kernel function is firstly validated, and the results are compared and discussed with those based on two single kernel functions. Second, the voltage prediction and SOH diagnosis results based on the short-term charging data are presented. Third, the influence of voltage noise is discussed. Finally, the features extracted from different voltage ranges are addressed to further assess the feasibility of the proposed method.

\section{A. Comparison with Different Kernel Functions}

To elucidate the advantages of the presented method, some commonly used kernel functions, including $\mathrm{RBF}$ and polynomial, are respectively applied. In addition, the mixed kernel function (MKF), in which the weights of RBF and polynomial kernel functions are both set as 0.5 , is also implemented. Fig. 3 shows the diagnosis results of the selected different kernel functions. We train the model using $50 \%$ of the cycle-SOH data of Bat. 1 and validate the model based on the remaining data of Bats 1 to 3. As shown in Fig. 3, the reference curve represents the actual $\mathrm{SOH}$ value which is obtained by calibration. The SA-MKF denotes the estimation results based on mixed kernel function and SA algorithm. It should be pointed out that the SOH in this study is denoted as the percentage of current capacity over the nominal value. The initial $\mathrm{SOH}$ is lower than $100 \%$ because of the operating environment and discharge conditions. In terms of Bat. 1, no matter what 
kernel functions are applied, all the absolute errors of $\mathrm{SOH}$ are restricted within $2 \%$, thus manifesting the feasibility of the proposed fixed size LS-SVM. However, the distribution of the proposed kernel function is relatively concentrated, compared to other three kernel functions. For Bat. 2, although the estimation results of RBF, polynomial and MKF can roughly track the reference value, their estimation errors are much larger than that of the SA-MKF, which is raised by the large local fluctuation of the estimation SOH. For Bat. 3, owing to the benefit from the mixed kernel function after optimization instead of the single kernel function, the estimation error of the SA-MKF fluctuates around zero while the prediction errors of other three kernel functions deviate far from zero. Therefore, we can infer that the proposed method with the optimal mixed kernel function shows a satisfactory generalization and guarantees high estimation precision.

The histograms of different kernel functions for Bat. 3 are compared in Fig. 3 (g) to (i) to further analyze the distribution of estimation errors. It can be indicated from these similar shapes that they have an approximate standard deviation. However, the number of samples near to zero is more for the presented kernel function and the error distribution is more concentrated, thus signifying that the mean error of the proposed method is less than those of the other three kernel functions. The standard deviation of the proposed, MKF, polynomial and RBF kernel functions are $0.44 \%, 0.41 \%, 0.44 \%$ and $0.56 \%$, respectively. The mean error of the proposed kernel function is $0.01 \%$. For the MKF, polynomial and RBF functions, their mean errors are respectively $0.33 \%, 0.4 \%$ and $0.11 \%$, obviously higher than that for the proposed kernel function. In conclusion, the proposed method is more accurate than other three kernel function-based estimation methods.

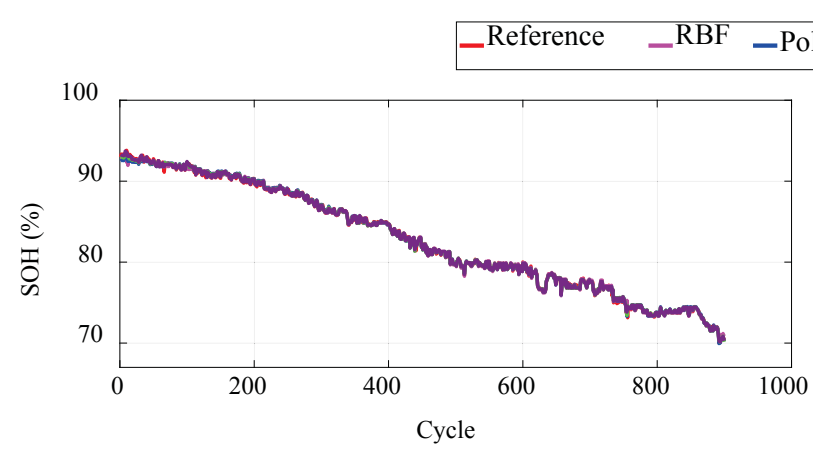

(a)

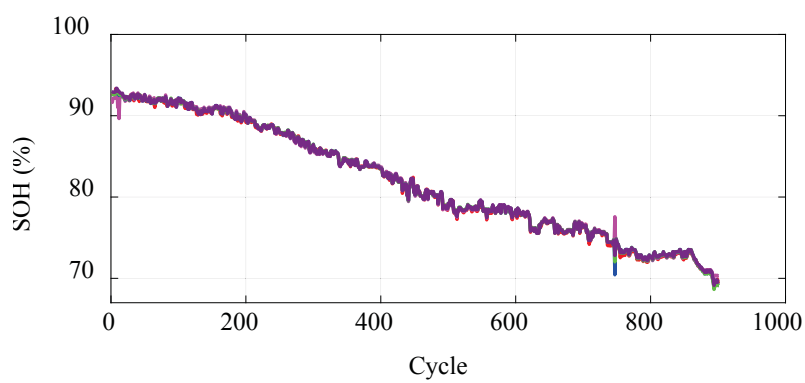

(c)



(b)

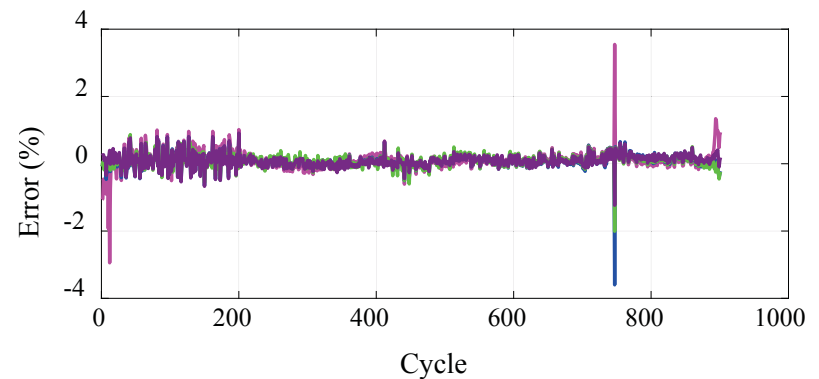

(d) 


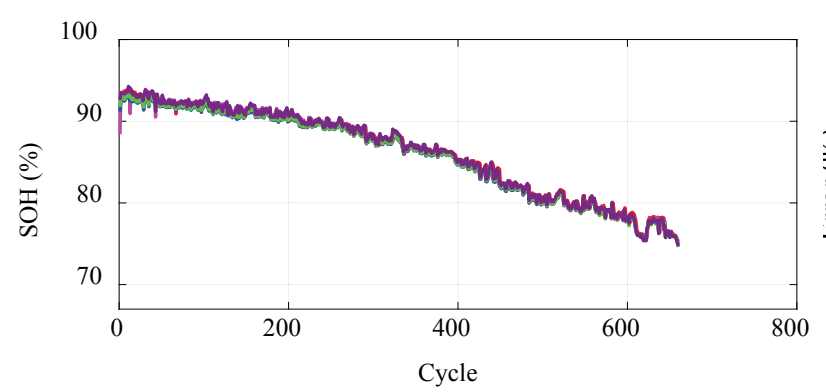

(e)

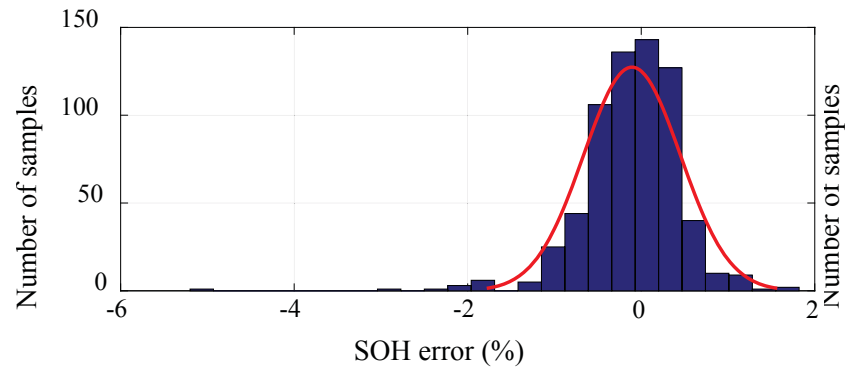

(g)

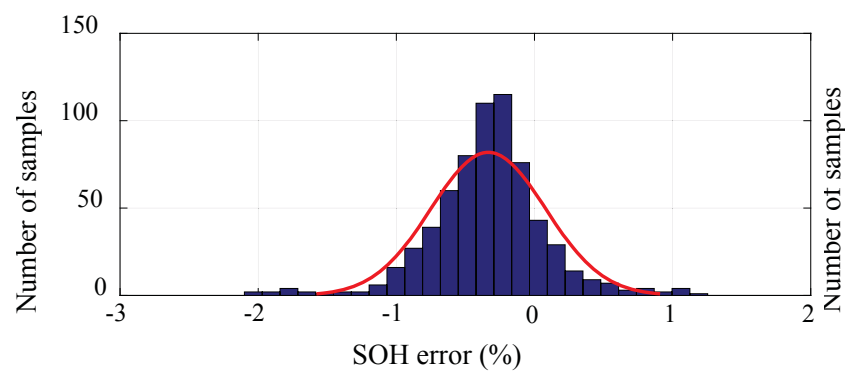

(i)

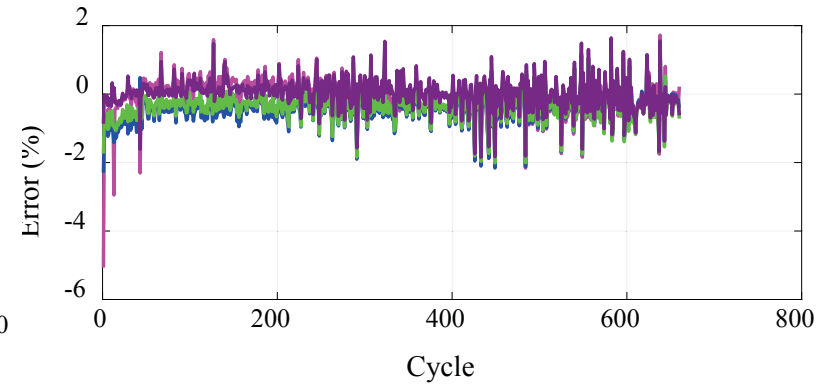

(f)

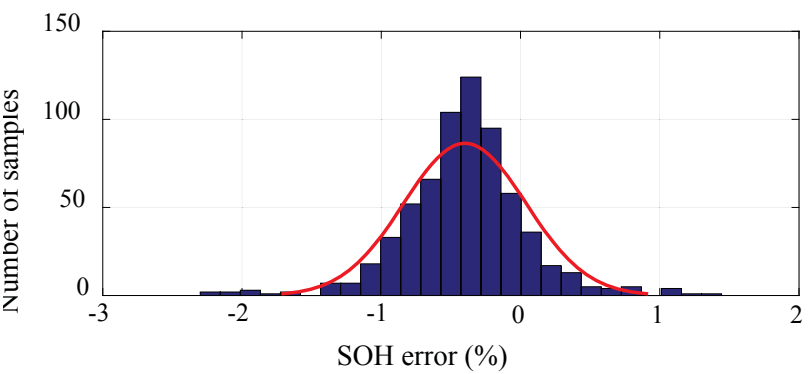

(h)

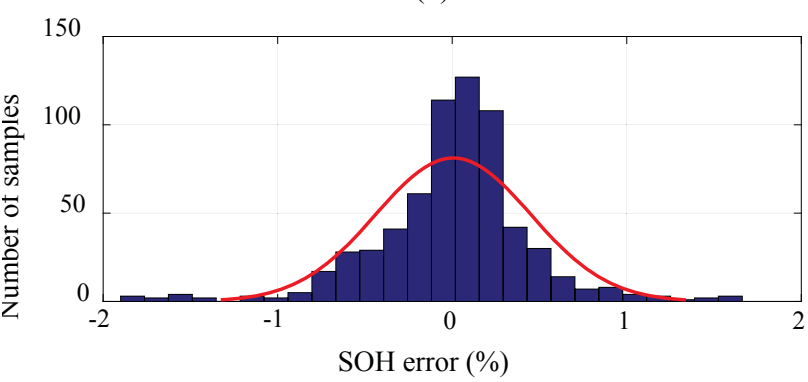

(j)

Fig. 3. SOH evolution with different kernel functions. (a) Bat. 1 prediction SOH results; (b) Bat. 1 prediction errors; (c) Bat. 2 prediction SOH results; (d) Bat. 2 prediction errors; (e) Bat. 3 prediction SOH results; (f) Bat. 3 prediction errors; (g) Histogram of RBF kernel function for Bat. 3; (h) Histogram of polynomial kernel function for Bat. 3; (i) Histogram of mixed kernel function for Bat. 3; (j) Histogram of SA-mixed kernel function for Bat. 3.

For the sake of assessing the diagnosis performances of the proposed method, the gained SOH values should be compared with the corresponding actual ones. The MAE, maximum absolute error (ME), and root mean square error (RMSE) are considered as the evaluation indicators for comparison. The error statistics based on the four kernel functions are listed in

Table I. It can be observed that the maximum ME, MAE and RMSE appear in Bat. 3 when the RBF kernel function is employed. While the ME of the proposed kernel function is lower than $1.9 \%$, about one-third of that of RBF. The MAE and RMSE of the proposed method for the three batteries are lower than $0.35 \%$ and $0.46 \%$, respectively. To sum up, the overall estimation performance of the proposed method outperforms that of the SVM algorithms with RBF, polynomial and the mixed kernel functions.

Table I. Statistical results with different kernel functions.

\begin{tabular}{llll}
\hline Test set & ME $(\%)$ & MAE $(\%)$ & RMSE (\%)
\end{tabular}




\begin{tabular}{lllllllllllll}
\hline Kernel & RBF & Poly. & MKF & SA- & RBF & Poly. & MKF & SA- & \multirow{2}{*}{ RBF } & Poly. & MKF & SA- \\
function & & & & & & \\
Bat. 1 & 2.23 & 1.66 & 1.63 & 1.42 & 0.19 & 0.17 & 0.138 & 0.10 & 0.27 & 0.24 & 0.20 & 0.15 \\
Bat. 2 & 4.11 & 3.60 & 2.89 & 1.51 & 0.25 & 0.17 & 0.19 & 0.16 & 0.38 & 0.26 & 0.26 & 0.21 \\
Bat. 3 & 5.83 & 3.53 & 3.43 & 1.90 & 0.54 & 0.53 & 0.46 & 0.32 & 0.74 & 0.67 & 0.62 & 0.46 \\
Mean & 4.06 & 2.93 & 2.65 & 1.61 & 0.33 & 0.29 & 0.26 & 0.19 & 0.46 & 0.39 & 0.36 & 0.27 \\
\hline
\end{tabular}

\section{B. SOH Validation Based on Short-Term Charging Voltage}

In this part, the forecast performance of the formed voltage prediction model is examined. As described in Section III, only one parameter needs to be set in the ELM. Herein, different numbers of neurons in the ELM model are utilized for model training. The RMSE of the voltage prediction and the running time are calculated to evaluate the performance of the ELM model. Fig. 4 demonstrates the mean RMSE, ME and running time as a function of neurons after training and test for ten times, where the training set is specified as the same (15 minutes of random charging data). Obviously, the calculation time of ELM increases significantly with the increase of the neuron number. When the number of neurons becomes more than 20, the RMSE and ME gradually converge to a stable value. It can also be clearly observed that once the number is more than 20 , increasing the number of neurons does not obviously improve the prediction performance but sacrifices the calculation time. From these results, we can find that the ELM does not lead to over-fit as the RMSE converges to a small value. Finally, $N_{\text {neuron }}$ is set to 23 after balancing the precision and the calculation intensity.

Based on the preset inputs, the prediction results of voltage at different cycles are demonstrated in Fig. 5 (a) and (b), which manifest that the ELM model can accomplish a satisfactory prognosis result and the forecasted voltages are quite close to the reference values. The statistic indicators of voltage prediction at different cycles on the training and testing data sets are compared in Table II. Note that we predict the charging voltage curve every 100 cycles, and it shows that no obvious decrease or increase of MAE and RMSE occur as the cycle number changes.

After selecting a suitable kernel function and determining a precise prediction voltage, we investigate the $\mathrm{SOH}$ diagnosis performance based on the predicted voltage curves. The battery SOH diagnosis results from these ten predicted voltages are depicted in Fig. 5 (c) and (d), and the statistical results are presented in Table II. It can be observed from these results that the output $\mathrm{SOH}$ of the presented method can track the reference curve precisely. We can see that only in the first cycle, the $\mathrm{SOH}$ estimation error is larger than $1 \%$, which can be attributed to the initial voltage prediction error. Certainly, the RMSE, ME and MAE of the predicted voltage in the first cycle are higher than those of other cycles and lead to a higher SOH error. Fig. 6 denotes a boxplot 
demonstrating the distribution of error statistical property across the cycle life test, wherein the red lines represent the median value and the red crosses indicate the outlier. As can be found, except a few abnormal points, the distribution of estimated results is all within the normal range. In summary, acceptable $\mathrm{SOH}$ diagnosis performance can be achieved by exerting a short-term charging data of just 15 minutes and extending the full charging voltage profile based on the ELM, and thus the feasibility of proposed algorithm is justified.

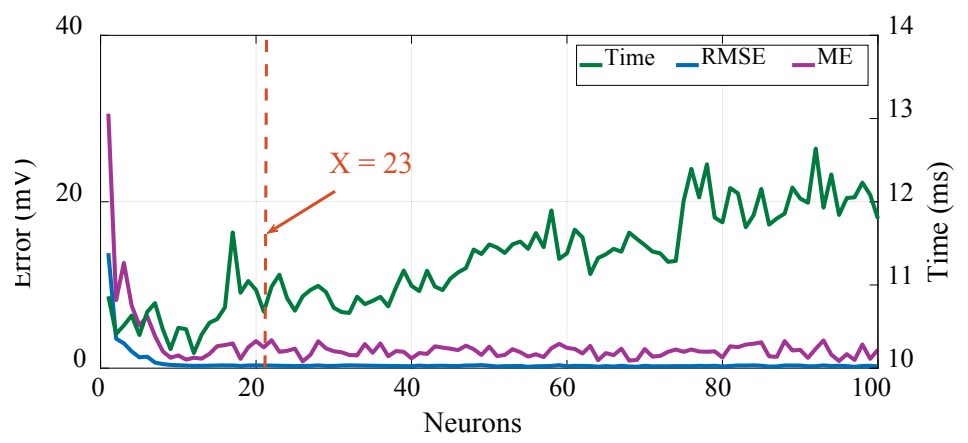

Fig. 4. Evaluation results of ELM model.

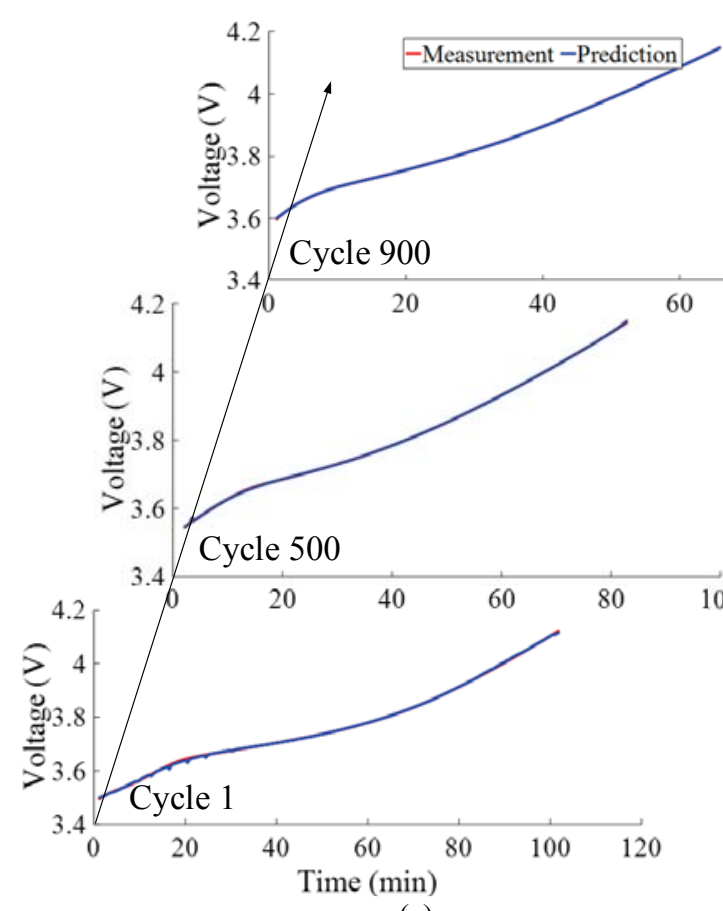

(a)

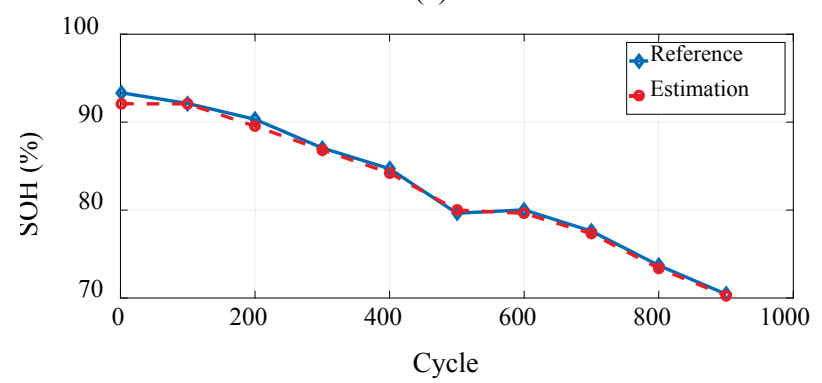

(c)

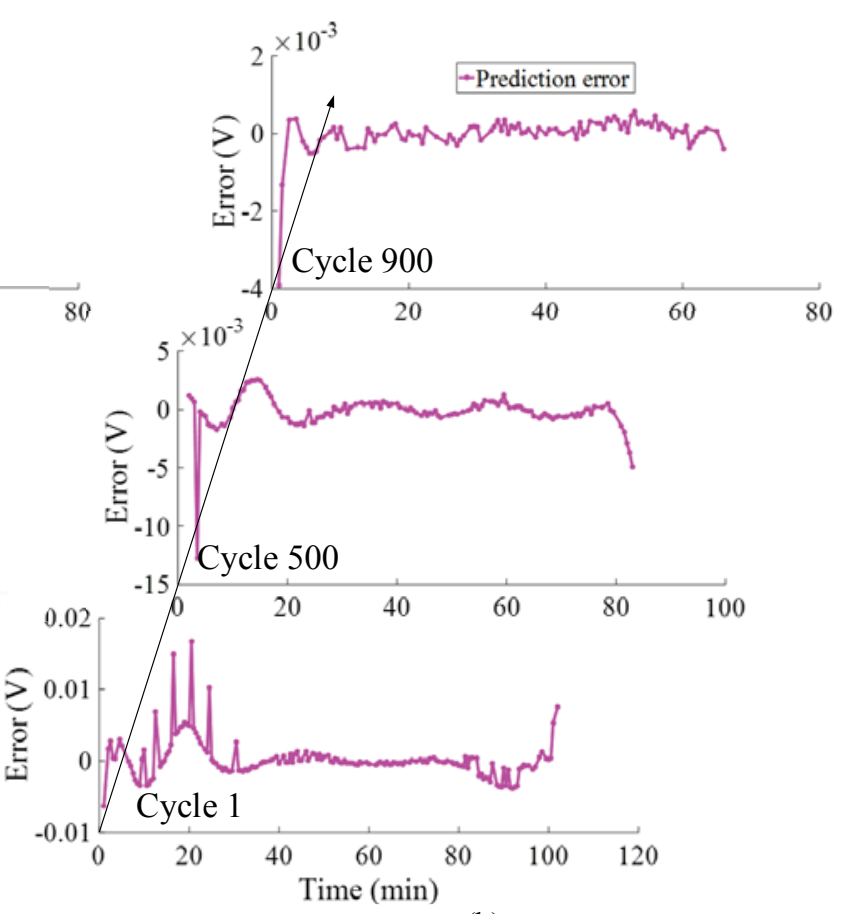

(b)

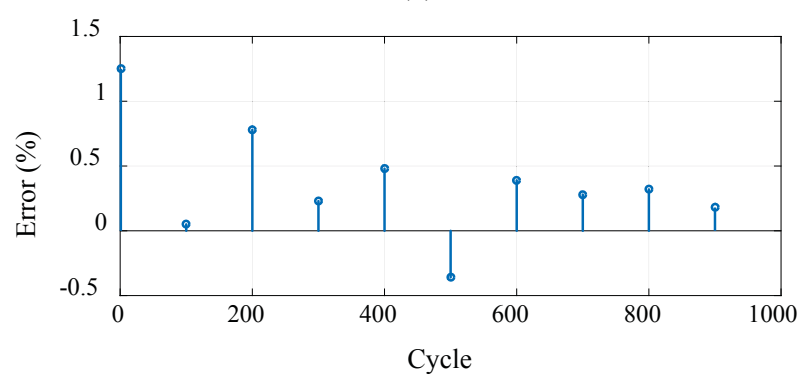

(d)

Fig. 5. Voltage and $\mathrm{SOH}$ prediction results based on the short-term charging voltage. (a) Voltage prediction results;

(b) Voltage prediction errors; (c) $\mathrm{SOH}$ prediction results; (d) $\mathrm{SOH}$ prediction errors. 


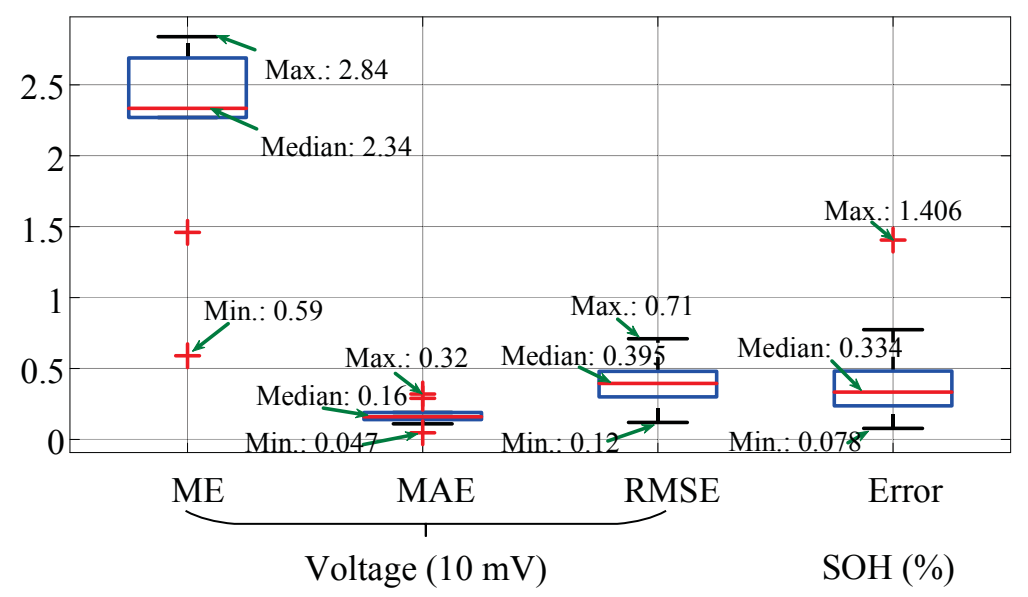

Fig. 6. Boxplot of the overall modeling performance.

Table II. Overall model performance.

\begin{tabular}{llllllllllll}
\hline \multicolumn{2}{l}{ Cycle number } & 1 & 100 & 200 & 300 & 400 & 500 & 600 & 700 & 800 & 900 \\
\hline \multirow{2}{*}{ Voltage } & ME & 2.84 & 2.37 & 2.27 & 1.46 & 2.64 & 2.69 & 2.74 & 2.28 & 2.30 & 0.59 \\
$(10 \mathrm{mV})$ & MAE & 0.32 & 0.29 & 0.16 & 0.14 & 0.17 & 0.19 & 0.16 & 0.11 & 0.15 & 0.047 \\
& RMSE & 0.71 & 0.53 & 0.35 & 0.29 & 0.41 & 0.48 & 0.38 & 0.30 & 0.42 & 0.12 \\
SOH (\%) & Error & 1.406 & 0.078 & 0.774 & 0.237 & 0.482 & 0.356 & 0.386 & 0.284 & 0.312 & 0.181 \\
\hline
\end{tabular}

\section{Influence of Voltage Noise}

To validate the robustness of presented $\mathrm{SOH}$ diagnosis method, the Gaussian noise, of which the mean and variance equal 0 and $0.01 \mathrm{~V}^{2}$, is imposed on the measured voltage under the CC charging experiment. Fig. 7 (a) depicts the voltage comparison at different cycles. Here, two noise setting modes are implemented. In mode 1, the training set is not disturbed by the noise and the Gaussian noise is overlapped into the testing set, which is used to validate the algorithm under the actual operation environment. In mode 2, the same Gaussian noise is added to the training and testing sets, indicating that the training and validation of the algorithm are all conducted in actual environment.

Fig. 7 shows the $\mathrm{SOH}$ estimation results under different scenarios of voltage noise. By comparing the estimation results of various noise conditions, we find that the training set without noise leads to better diagnosis performance, and in contrast, the training set with voltage noise highlights the inferior estimation results. As can be observed from Table III, the prediction results based on mode 1 are more accurate. Concretely, the ME based on mode 1 is larger; even so, the ME is still less than $1.8 \%$. The similar values of RMSE and MAE for these two modes indicate that the proposed feature extraction and SOH diagnosis method show strong robustness. Even when the noises are imposed in the training and testing sets, a preferable 
estimation result can be still achieved by the proposed method, and one main attribution is that the proposed feature selection method characterizes strong anti-interference ability.

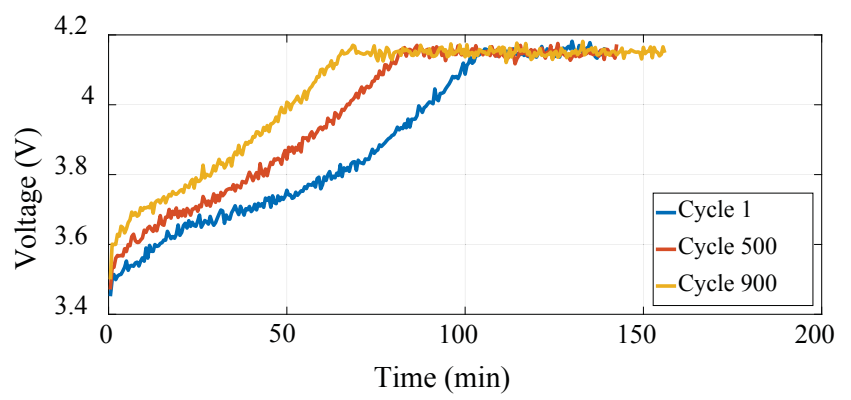

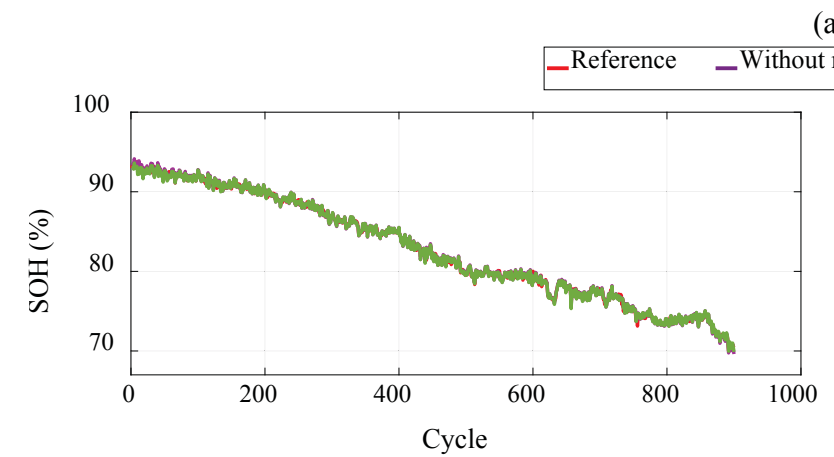

(b)

(a)

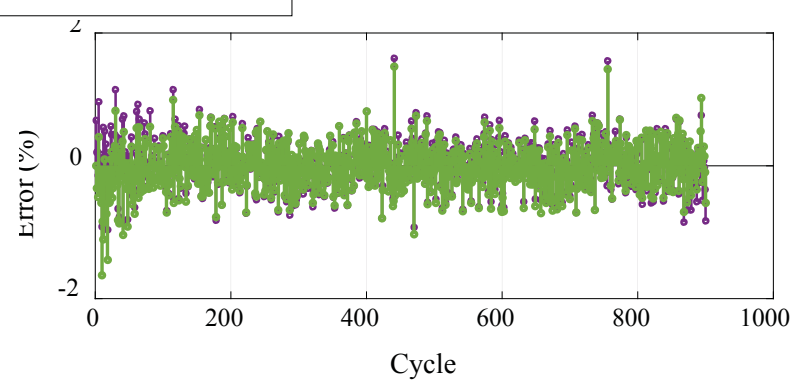

(c)

Fig. 7. SOH estimation results with voltage noise. (a) Voltage profiles with noise; (b) SOH prediction results; (c) SOH prediction errors.

Table III. Errors of SOH estimations under voltage noise.

\begin{tabular}{llllll}
\hline Group & Training set & Testing set & ME (\%) & MAE (\%) & RMSE (\%) \\
\hline 1 & Without noise & \multirow{2}{*}{ With noise } & 1.69 & 0.27 & 0.35 \\
2 & With noise & & 1.77 & 0.27 & 0.35 \\
\hline
\end{tabular}

\section{Influence of Voltage Range}

As demonstrated in Section III, a specific segmenting method of voltage curve should be firstly designated for feature generation and model training. In this part, three different ranges, as presented in Table IV, are considered for the feature generation. They are selected to validate how the voltage range will influence the diagnosis performance of $\mathrm{SOH}$. It is noteworthy that in Range 3, there exist only two feature variables, both of which are overlapped. Since we have reselected the input features, the model needs to be trained again, and the model parameters are also optimized by the SA algorithm. To facilitate comparison with previous diagnosis results, $50 \%$ data of Bat. 1 is applied to train the model, and other data sets are used to evaluate the model performance. The SOH diagnosis results of unfamiliar data sets by the proposed kernel function method with different voltage ranges are shown in Fig. 8. It can be observed that the proposed kernel function approach can strictly follow the $\mathrm{SOH}$ decline tendency for all the tested batteries with various voltage ranges. 
Table IV. Segmentation range of voltage curve.

\begin{tabular}{llll}
\hline & Segment 1 & Segment 2 & Segment 3 \\
\hline Range 1 & $(2.753 .65]$ & $(2.753 .65]$ & $(4.054 .15]$ \\
Range 2 & $(2.753 .70]$ & $(3.73 .65]$ & $(4.14 .15]$ \\
Range 3 & $(2.753 .90]$ & $(2.94 .14]$ & \\
\hline
\end{tabular}

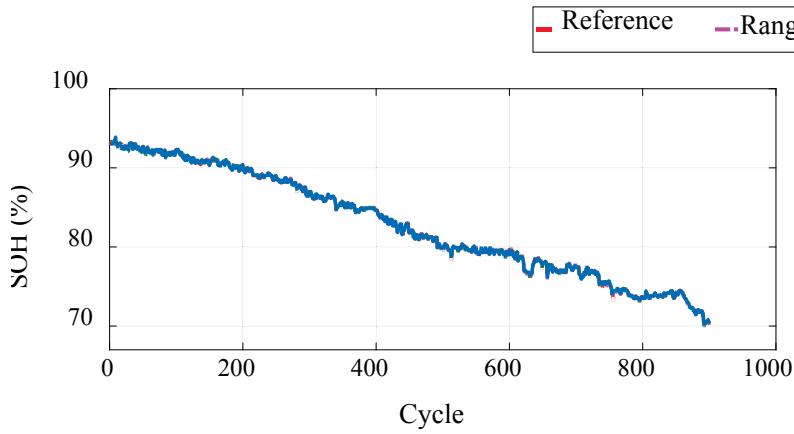

(a)

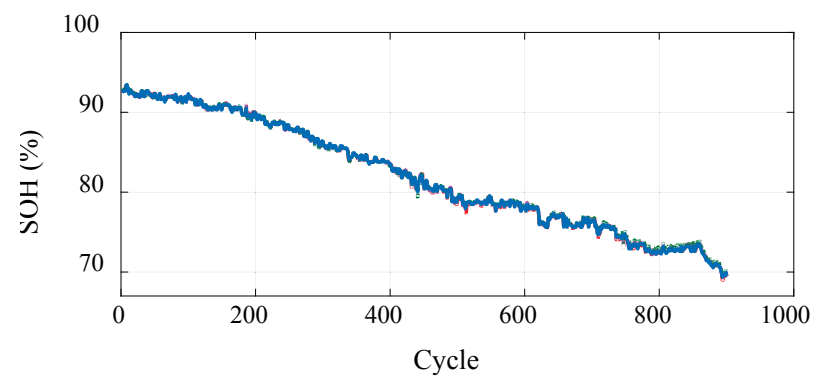

(c)

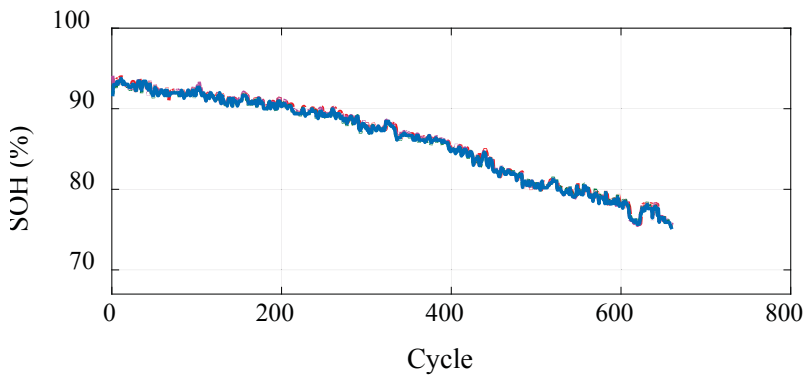

(e)



(b)

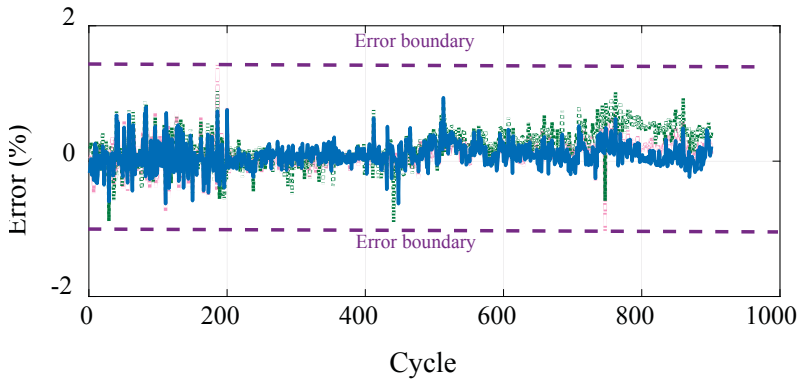

(d)

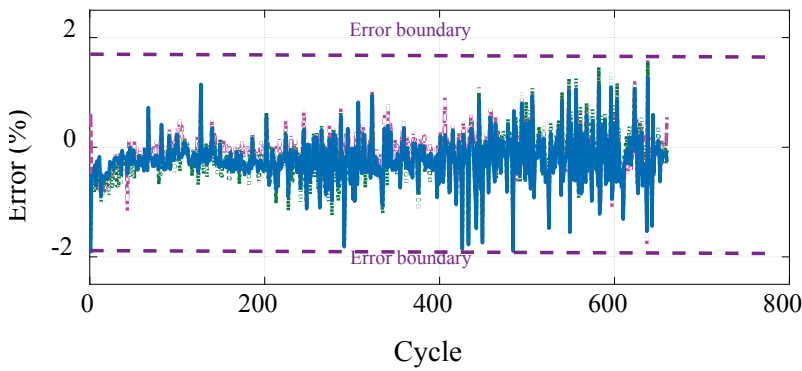

(f)

Fig. 8. SOH diagnosis results under different voltage regions. (a) Bat. 1 prediction $\mathrm{SOH}$; (b) Bat. 1 prediction errors;

(c) Bat. 2 prediction results; (d) Bat. 2 prediction errors; (e) Bat. 3 prediction results; (f) Bat. 3 prediction errors.

To further assess the diagnosis capability of each voltage range, the statistics results for all batteries are compared in Table V. The least ME, MAE and RMSE are distinguished as bold characters for clearer comparison. According to Table V, for all the cells, different voltage ranges manifest the varying prediction precision. It is apparent that the presented method achieved the optimal performance with the input data under Range 1. Although the results of Range 1 on Bat. 2 are not as good as those based on Range 3, from the perspective of global results, the estimated result of Range 1 is still better than that of Range 3 , with the mean ME, RMSE and MAE as $1.69 \%, 0.19 \%$ and $0.278 \%$, respectively. By this manner, the feasibility of proposed voltage range selection and $\mathrm{SOH}$ diagnosis algorithm is justified. 
Table V. Comparison results under different voltage regions.

\begin{tabular}{llllllllll}
\hline Battery & ME (\%) & \multicolumn{3}{c}{ MAE (\%) } \\
\cline { 2 - 10 } Num. & Range 1 & Range 2 & Range 3 & Range 1 & Range 2 & Range 3 & Range 1 & Range 2 & Range 3 \\
\hline 1 & $\mathbf{1 . 2 6}$ & 1.37 & 1.33 & $\mathbf{0 . 1 1}$ & 0.15 & 0.12 & $\mathbf{0 . 1 6}$ & 0.19 & 0.17 \\
2 & 1.93 & 1.44 & $\mathbf{0 . 9 5}$ & 0.17 & 0.28 & $\mathbf{0 . 1 5}$ & 0.24 & 0.36 & $\mathbf{0 . 2 1}$ \\
3 & $\mathbf{1 . 8 7}$ & 1.85 & 1.95 & $\mathbf{0 . 3 0}$ & 0.37 & 0.36 & $\mathbf{0 . 4 3}$ & 0.49 & 0.47 \\
mean & 1.69 & 1.55 & $\mathbf{1 . 4 1}$ & $\mathbf{0 . 1 9}$ & 0.27 & 0.21 & $\mathbf{0 . 2 7 8}$ & 0.35 & 0.283 \\
\hline
\end{tabular}

\section{CONCLUSIONS}

In this study, an online state of health diagnosis approach is developed based on short-term measurement data that is acquired under the constant current charging mode. The proposed method only requires random discontinuous charging data with a length of 15 minutes to predict a complete constant current charging voltage curve by the extreme learning machine, thus enabling more flexible battery state of health diagnosis. The fixed size least squares support vector machine model based on a mixed kernel function is established to capture the nonlinear characteristics of state of health based on the feature variables extracted from the predicted voltage without pre-processing. The illustrative results confirm that the developed mixed kernel function based algorithm outperforms the algorithm with the single kernel functions in terms of state of health diagnosis accuracy and generality. The validation experiments highlight that the proposed state of health diagnosis method features strong anti-interference capability. Moreover, the results in different voltage ranges also indicate that the proposed method is accurate, reliable and effective. All of the experimental results demonstrate that the maximum absolute error of the proposed method can be restricted within $2 \%$.

To the best of authors' knowledge, this is the first attempt of directly coupling the voltage prediction and state of health diagnosis into a unified framework. It can be inferred that the developed method has the potential for practical application. Nonetheless, there still exists some improvement space in future research with respect to calculation speed and prediction accuracy. Furthermore, lithium-ion battery degradation mechanism and state of health prediction from the perspective of electrochemistry under varying ambient temperatures and different current rates should also be investigated in-depth in our future work.

\section{ACKNOWLEDGMENTS}

This work was supported in part by the National Natural Science Foundation of China (No. 61763021 and No. 51775063), in part by the National Key R\&D Program of China (No. 2018YFB0104000), and in part 
by the EU-funded Marie Skłodowska-Curie Individual Fellowships Project under Grant 845102-HOEMEV-

H2020-MSCA-IF-2018.

\section{REFERENCES}

[1] Chen Z, Shu X, Xiao R, Yan W, Liu Y, Shen J. Optimal Charging Strategy Design for Lithium - Ion Batteries Considering Minimization of Temperature Rise and Energy Loss. International Journal of Energy Research. 2019;43(9):4344-58.

[2] Tang X, Zou C, Yao K, Lu J, Xia Y, Gao F. Aging Trajectory Prediction for Lithium-Ion Batteries Via Model Migration and Bayesian Monte Carlo Method. Applied Energy. 2019;254:113591.

[3] Jiang Y, Jiang J, Zhang C, Zhang W, Gao Y, Li N. State of Health Estimation of Second-Life Lifepo4 Batteries for Energy Storage Applications. Journal of cleaner production. 2018;205:754-62.

[4] Song Y, Liu D, Liao H, Peng Y. A Hybrid Statistical Data-Driven Method for on-Line Joint State Estimation of Lithium-Ion Batteries. Applied Energy. 2020;261:114408.

[5] Hu X, Xu L, Lin X, Pecht M. Battery Lifetime Prognostics. Joule. 2020.

[6] Cui Y, Zuo P, Du C, Gao Y, Yang J, Cheng X, Ma Y, Yin G. State of Health Diagnosis Model for Lithium Ion Batteries Based on Real-Time Impedance and Open Circuit Voltage Parameters Identification Method. Energy. 2018;144:647-56.

[7] Lipu MH, Hannan M, Hussain A, Hoque M, Ker PJ, Saad MHM, Ayob A. A Review of State of Health and Remaining Useful Life Estimation Methods for Lithium-Ion Battery in Electric Vehicles: Challenges and Recommendations. Journal of cleaner production. 2018;205:115-33.

[8] Shen P, Ouyang M, Lu L, Li J, Feng X. The Co-Estimation of State of Charge, State of Health, and State of Function for Lithium-Ion Batteries in Electric Vehicles. IEEE Transactions on vehicular technology. 2017;67(1):92-103.

[9] Chen C, Xiong R, Shen W. A Lithium-Ion Battery-in-the-Loop Approach to Test and Validate Multiscale Dual H Infinity Filters for State-of-Charge and Capacity Estimation. IEEE Transactions on power Electronics. 2017;33(1):332-42.

[10] Tian J, Xiong R, Yu Q. Fractional-Order Model-Based Incremental Capacity Analysis for Degradation State Recognition of Lithium-Ion Batteries. IEEE Transactions on Industrial Electronics. 2018;66(2):1576-84.

[11] Zou Y, Hu X, Ma H, Li SE. Combined State of Charge and State of Health Estimation over Lithium-Ion Battery Cell Cycle Lifespan for Electric Vehicles. Journal of Power Sources. 2015;273:793-803.

[12] Feng Y, Xue C, Han Q-L, Han F, Du J. Robust Estimation for State-of-Charge and State-of-Health of Lithium-Ion Batteries Using Integral-Type Terminal Sliding-Mode Observers. IEEE Transactions on Industrial Electronics. 2019.

[13] Liu D, Yin X, Song Y, Liu W, Peng Y. An on-Line State of Health Estimation of Lithium-Ion Battery Using Unscented Particle Filter. Ieee Access. 2018;6:40990-1001.

[14] El Mejdoubi A, Oukaour A, Chaoui H, Gualous H, Sabor J, Slamani Y. State-of-Charge and State-of-Health LithiumIon Batteries' Diagnosis According to Surface Temperature Variation. IEEE Transactions on Industrial Electronics. 2015;63(4):2391-402.

[15] Li Y, Abdel-Monem M, Gopalakrishnan R, Berecibar M, Nanini-Maury E, Omar N, van den Bossche P, Van Mierlo J. A Quick on-Line State of Health Estimation Method for Li-Ion Battery with Incremental Capacity Curves Processed by Gaussian Filter. Journal of Power Sources. 2018;373:40-53.

[16] Chen Z, Xue Q, Xiao R, Liu Y, Shen J. State of Health Estimation for Lithium-Ion Batteries Based on Fusion of Autoregressive Moving Average Model and Elman Neural Network. IEEE Access. 2019;7:102662-78.

[17] Li X, Yuan C, Li X, Wang Z. State of Health Estimation for Li-Ion Battery Using Incremental Capacity Analysis and Gaussian Process Regression. Energy. 2020;190:116467.

[18] Chen Z, Sun M, Shu X, Xiao R, Shen J. Online State of Health Estimation for Lithium-Ion Batteries Based on Support Vector Machine. Applied Sciences. 2018;8(6):925.

[19] Liu Y, Li J, Chen Z, Qin D, Zhang Y. Research on a Multi-Objective Hierarchical Prediction Energy Management Strategy for Range Extended Fuel Cell Vehicles. Journal of Power Sources. 2019;429:55-66.

[20] Guo P, Cheng Z, Yang L. A Data-Driven Remaining Capacity Estimation Approach for Lithium-Ion Batteries Based on Charging Health Feature Extraction. Journal of Power Sources. 2019;412:442-50.

[21] Lu C, Tao L, Fan H. Li-Ion Battery Capacity Estimation: A Geometrical Approach. Journal of power sources. 2014;261:141-7.

[22] Hu C, Jain G, Schmidt C, Strief C, Sullivan M. Online Estimation of Lithium-Ion Battery Capacity Using Sparse Bayesian Learning. Journal of Power Sources. 2015;289:105-13.

[23] Meng J, Cai L, Stroe D-I, Luo G, Sui X, Teodorescu R. Lithium-Ion Battery State-of-Health Estimation in Electric Vehicle Using Optimized Partial Charging Voltage Profiles. Energy. 2019;185:1054-62.

[24] Richardson RR, Birkl CR, Osborne MA, Howey DA. Gaussian Process Regression for in Situ Capacity Estimation of Lithium-Ion Batteries. IEEE Transactions on Industrial Informatics. 2018;15(1):127-38. 
[25] Berecibar M, Devriendt F, Dubarry M, Villarreal I, Omar N, Verbeke W, Van Mierlo J. Online State of Health Estimation on Nmc Cells Based on Predictive Analytics. Journal of Power Sources. 2016;320:239-50.

[26] Feng X, Weng C, He X, Han X, Lu L, Ren D, Ouyang M. Online State-of-Health Estimation for Li-Ion Battery Using Partial Charging Segment Based on Support Vector Machine. IEEE Transactions on Vehicular Technology. 2019;68(9):8583-92.

[27] Wang F-K, Mamo T. A Hybrid Model Based on Support Vector Regression and Differential Evolution for Remaining Useful Lifetime Prediction of Lithium-Ion Batteries. Journal of Power Sources. 2018;401:49-54.

[28] Yang D, Wang Y, Pan R, Chen R, Chen Z. State-of-Health Estimation for the Lithium-Ion Battery Based on Support Vector Regression. Applied Energy. 2018;227:273-83.

[29] Pan H, Lü Z, Wang H, Wei H, Chen L. Novel Battery State-of-Health Online Estimation Method Using Multiple Health Indicators and an Extreme Learning Machine. Energy. 2018;160:466-77.

[30] Huang G-B, Zhu Q-Y, Siew C-K. Extreme Learning Machine: Theory and Applications. Neurocomputing. 2006;70(1-3):489-501.

[31] Suykens JA, De Brabanter J, Lukas L, Vandewalle J. Weighted Least Squares Support Vector Machines: Robustness and Sparse Approximation. Neurocomputing. 2002;48(1-4):85-105.

[32] Inoue T, Abe S. Fuzzy Support Vector Machines for Pattern Classification. Conference Fuzzy Support Vector Machines for Pattern Classification, vol. 2. IEEE, p. 1449-54.

[33] Fung GM, Mangasarian OL. Multicategory Proximal Support Vector Machine Classifiers. Machine learning. 2005;59(1-2):77-97.

[34] AK SJ. Least Squares Support Vector Machines: World Scientific, 2002.

[35] Zhu B, Shi X, Chevallier J, Wang P, Wei YM. An Adaptive Multiscale Ensemble Learning Paradigm for Nonstationary and Nonlinear Energy Price Time Series Forecasting. Journal of Forecasting. 2016;35(7):633-51.

[36] Zhu B, Ye S, Jiang M, Wang P, Wu Z, Xie R, Chevallier J, Wei Y-M. Achieving the Carbon Intensity Target of China: A Least Squares Support Vector Machine with Mixture Kernel Function Approach. Applied energy. 2019;233:196-207.

[37] Vapnik V. The Nature of Statistical Learning Theory: Springer science \& business media, 2013.

[38] Yun L, Maddila S, Gao L, Peng X, Niu X, Garg A, Chin CMM. An Integrated Framework for Minimization of Inter Lithium - Ion Cell Temperature Differences and the Total Volume of the Cell of Battery Pack for Electric Vehicles. Energy Storage. 2019;1(2):e41. 